\title{
A pinching theorem for the first eigenvalue of the Laplacian on hypersurfaces of the Euclidean space
}

\author{
Bruno Colbois and Jean-François Grosjean*
}

\begin{abstract}
In this paper, we give pinching theorems for the first nonzero eigenvalue $\lambda_{1}(M)$ of the Laplacian on the compact hypersurfaces of the Euclidean space. Indeed, we prove that if the volume of $M$ is 1 then, for any $\varepsilon>0$, there exists a constant $C_{\varepsilon}$ depending on the dimension $n$ of $M$ and the $L_{\infty}$-norm of the mean curvature $H$, so that if the $L_{2 p}$-norm $\|H\|_{2 p}(p \geq 2)$ of $H$ satisfies $n\|H\|_{2 p}^{2}-C_{\varepsilon}<\lambda_{1}(M)$, then the Hausdorff-distance between $M$ and a round sphere of radius $\left(n / \lambda_{1}(M)\right)^{1 / 2}$ is smaller than $\varepsilon$. Furthermore, we prove that if $C$ is a small enough constant depending on $n$ and the $L_{\infty}$-norm of the second fundamental form, then the pinching condition $n\|H\|_{2 p}^{2}-C<\lambda_{1}(M)$ implies that $M$ is diffeomorphic to an $n$-dimensional sphere.
\end{abstract}

Mathematics Subject Classification (2000). 53A07, 53C21.

Keywords. Spectrum, Laplacian, pinching results, hypersurfaces.

\section{Introduction and preliminaries}

Let $\left(M^{n}, g\right)$ be a compact, connected and oriented $n$-dimensional Riemannian manifold without boundary isometrically immersed by $\phi$ into the $n+1$-dimensional euclidean space $\left(\mathbb{R}^{n+1}\right.$, can) (i.e. $\phi^{\star}$ can $=g$ ). A well-known inequality due to Reilly ([11]) gives an extrinsic upper bound for the first nonzero eigenvalue $\lambda_{1}(M)$ of the Laplacian of $\left(M^{n}, g\right)$ in terms of the square of the length of the mean curvature. Indeed, we have

$$
\lambda_{1}(M) \leq \frac{n}{V(M)} \int_{M}|H|^{2} d v
$$

where $d v$ and $V(M)$ denote respectively the Riemannian volume element and the volume of $\left(M^{n}, g\right)$. Moreover the equality holds if and only if $\left(M^{n}, g\right)$ is a geodesic hypersphere of $\mathbb{R}^{n+1}$.

\footnotetext{
${ }^{*}$ Supported by European Commission through Human Potential Programme.
} 
By using Hölder inequality, we obtain some other similar estimates for the $L_{2 p^{-}}$ norm $(p \geq 1)$ with $H$ denoted by $\|H\|_{2 p}^{2}$

$$
\lambda_{1}(M) \leq \frac{n}{V(M)^{1 / p}}\|H\|_{2 p}^{2},
$$

and as for the inequality (1), the equality case is characterized by the geodesic hyperspheres of $\mathbb{R}^{n+1}$.

A first natural question is to know if there exists a pinching result as the one we state now: does a constant $C$ depending on minimum geometric invariants exist so that if we have the pinching condition

$$
\frac{n}{V(M)^{1 / p}}\|H\|_{2 p}^{2}-C<\lambda_{1}(M)
$$

then $M$ is close to a sphere in a certain sense?

Such questions are known for the intrinsic lower bound of Lichnerowicz-Obata ([9]) of $\lambda_{1}(M)$ in terms of the lower bound of the Ricci curvature (see [4], [8], [10]). Other pinching results have been proved for Riemannian manifolds with positive Ricci curvature, with a pinching condition on the $n+1$-st eigenvalue ([10]), the diameter ([5], [8], [15]), the volume or the radius (see for instance [2] and [3]).

For instance, S. Ilias proved in [8] that there exists $\varepsilon$ depending on $n$ and an upper bound of the sectional curvature so that if the Ricci curvature Ric of $M$ satisfies Ric $\geq n-1$ and $\lambda_{1}(M) \leq \lambda_{1}\left(\mathbb{S}^{n}\right)+\varepsilon$, then $M$ is homeomorphic to $\mathbb{S}^{n}$.

In this article, we investigate the case of hypersurfaces where, as far as we know, very little is known about pinching and stability results (see however [12], [13]).

More precisely, in our paper, the hypothesis made in [8] that $M$ has a positive Ricci curvature is replaced by the fact that $M$ is isometrically immersed as a hypersurface in $\mathbb{R}^{n+1}$, and the bound on the sectional curvature by an $L^{\infty}$-bound on the mean curvature or on the second fundamental form. Note that we do not know if such bounds are sharp, or if a bound on the $L^{q}$-norm (for some $q$ ) of the mean curvature would be enough.

We get the following results

Theorem 1.1. Let $\left(M^{n}, g\right)$ be a compact, connected and oriented $n$-dimensional Riemannian manifold without boundary isometrically immersed by $\phi$ in $\mathbb{R}^{n+1}$. Assume that $V(M)=1$ and let $x_{0}$ be the center of mass of $M$. Then for any $p \geq 2$ and for any $\varepsilon>0$, there exists a constant $C_{\varepsilon}$ depending only on $n, \varepsilon>0$ and on the $L_{\infty}$-norm of $H$ so that if

$$
\left(\mathrm{P}_{C_{\varepsilon}}\right) \quad n\|H\|_{2 p}^{2}-C_{\varepsilon}<\lambda_{1}(M)
$$

then the Hausdorff-distance $d_{H}$ of $M$ to the sphere $S\left(x_{0}, \sqrt{\frac{n}{\lambda_{1}(M)}}\right)$ of center $x_{0}$ and radius $\sqrt{\frac{n}{\lambda_{1}(M)}}$ satisfies $d_{H}\left(\phi(M), S\left(x_{0}, \sqrt{\frac{n}{\lambda_{1}(M)}}\right)\right)<\varepsilon$. 
We recall that the Hausdorff-distance between two compact subsets $A$ and $B$ of a metric space is given by

$$
d_{H}(A, B)=\inf \left\{\eta \mid V_{\eta}(A) \supset B \text { and } V_{\eta}(B) \supset A\right\}
$$

where for any subset $A, V_{\eta}(A)$ is the tubular neighborhood of $A$ defined by $V_{\eta}(A)=$ $\{x \mid \operatorname{dist}(x, A)<\eta\}$.

Remark. We will see in the proof that $C_{\varepsilon}\left(n,\|H\|_{\infty}\right) \rightarrow 0$ when $\|H\|_{\infty} \rightarrow \infty$ or $\varepsilon \rightarrow 0$.

In fact the previous theorem is a consequence of the above definition and the following theorem

Theorem 1.2. Let $\left(M^{n}, g\right)$ be a compact, connected and oriented n-dimensional Riemannian manifold without boundary isometrically immersed by $\phi$ in $\mathbb{R}^{n+1}$. Assume that $V(M)=1$ and let $x_{0}$ be the center of mass of $M$. Then for any $p \geq 2$ and for any $\varepsilon>0$, there exists a constant $C_{\varepsilon}$ depending only on $n, \varepsilon>0$ and on the $L_{\infty}$-norm of $H$ so that if

$$
\left(\mathrm{P}_{C_{\varepsilon}}\right) \quad n\|H\|_{2 p}^{2}-C_{\varepsilon}<\lambda_{1}(M)
$$

then

$$
\begin{aligned}
& \text { (1) } \phi(M) \subset B\left(x_{0}, \sqrt{\frac{n}{\lambda_{1}(M)}}+\varepsilon\right) \backslash B\left(x_{0}, \sqrt{\frac{n}{\lambda_{1}(M)}}-\varepsilon\right) \text {; } \\
& \text { (2) } B(x, \varepsilon) \cap \phi(M) \neq \varnothing \text { for all } x \in S\left(x_{0}, \sqrt{\frac{n}{\lambda_{1}(M)}}\right) \text {. }
\end{aligned}
$$

In the following theorem, if the pinching is strong enough, with a control on $n$ and the $L_{\infty}$-norm of the second fundamental form, we obtain that $M$ is diffeomorphic to a sphere and even almost isometric with a round sphere in a sense we will make precise.

Theorem 1.3. Let $\left(M^{n}, g\right)$ be a compact, connected and oriented n-dimensional Riemannian manifold $(n \geq 2)$ without boundary isometrically immersed by $\phi$ in $\mathbb{R}^{n+1}$. Assume that $V(M)=1$. Then for any $p \geq 2$, there exists a constant $C$ depending only on $n$ and the $L_{\infty}$-norm of the second fundamental form $B$ so that if

$$
n\|H\|_{2 p}^{2}-C<\lambda_{1}(M) .
$$

Then $M$ is diffeomorphic to $\mathbb{S}^{n}$.

More precisely, there exists a diffeomorphism $F$ from $M$ into the sphere $\mathbb{S}^{n}\left(\sqrt{\frac{n}{\lambda_{1}(M)}}\right)$ of radius $\sqrt{\frac{n}{\lambda_{1}(M)}}$ which is a quasi-isometry. Namely, for any $\theta$, 
$0<\theta<1$, there exists a constant $C$ depending only on $n$, the $L_{\infty}$-norm of $B$ and $\theta$, so that the pinching condition $\left(\mathrm{P}_{C}\right)$ implies

$$
\left.|| d F_{x}(u)\right|^{2}-1 \mid \leq \theta
$$

for any $x \in M$ and $u \in T_{x} M$ so that $|u|=1$.

Now we will give some preliminaries for the proof of these theorems. Throughout the paper, we consider a compact, connected and oriented $n$-dimensional Riemannian manifold $\left(M^{n}, g\right)$ without boundary isometrically immersed by $\phi$ into $\left(\mathbb{R}^{n+1}\right.$, can $)$ (i.e. $\phi^{\star} c a n=g$ ). Let $v$ be the outward normal vector field. Then the second fundamental form of the immersion will be defined by $B(X, Y)=\left\langle\nabla_{X}^{0} \nu, Y\right\rangle$, where $\nabla^{0}$ and $\langle$,$\rangle are respectively the Riemannian connection and the inner product of \mathbb{R}^{n+1}$. Moreover the mean curvature $H$ will be given by $H=(1 / n)$ trace $(B)$.

Now let $\partial_{i}$ be an orthonormal frame of $\mathbb{R}^{n+1}$ and let $x_{i}: \mathbb{R}^{n+1} \rightarrow \mathbb{R}$ be the associated component functions. Putting $X_{i}=x_{i} \circ \phi$, a straightforward calculation shows us that

$$
B \otimes v=-\sum_{i \leq n+1} \nabla d X_{i} \otimes \partial_{i}
$$

and

$$
n H v=\sum_{i \leq n+1} \Delta X_{i} \partial_{i},
$$

where $\nabla$ and $\Delta$ denote respectively the Riemannian connection and the LaplaceBeltrami operator of $\left(M^{n}, g\right)$. On the other hand, we have the well-known formula

$$
\frac{1}{2} \Delta|X|^{2}=n H\langle v, X\rangle-n
$$

where $X$ is the position vector given by $X=\sum_{i \leq n+1} X_{i} \partial_{i}$.

We recall that to prove the Reilly inequality, we use the functions $X_{i}$ as test functions (cf. [11]). Indeed, doing a translation if necessary, we can assume that $\int_{M} X_{i} d v=0$ for all $i \leq n+1$ and we can apply the variational characterization of $\lambda_{1}(M)$ to $X_{i}$. If the equality holds in (1) or (2), then the functions are nothing but eigenfunctions of $\lambda_{1}(M)$ and from the Takahashi Theorem ([14]) $M$ is immersed isometrically in $\mathbb{R}^{n+1}$ as a geodesic sphere of radius $\sqrt{\frac{n}{\lambda_{1}(M)}}$.

Throughout the paper we use some notations. From now on, the inner product and the norm induced by $g$ and can on a tensor $T$ will be denoted respectively by $\langle$,$\rangle and ||^{2}$, and the $L_{p}$-norm will be given by

$$
\|T\|_{p}=\left(\int_{M}|T|^{p} d v\right)^{1 / p}
$$


and

$$
\|T\|_{\infty}=\sup _{M}|T| .
$$

We end these preliminaries by a convenient result.

Lemma 1.1. Let $\left(M^{n}, g\right)$ be a compact, connected and oriented $n$-dimensional Riemannian manifold $(n \geq 2)$ without boundary isometrically immersed by $\phi$ in $\mathbb{R}^{n+1}$. Assume that $V(M)=1$. Then there exist constants $c_{n}$ and $d_{n}$ depending only on $n$ so that for any $p \geq 2$, if $\left(\mathrm{P}_{C}\right)$ is true with $C<c_{n}$ then

$$
\frac{n}{\lambda_{1}(M)} \leq d_{n}
$$

Proof. We recall the standard Sobolev inequality (cf. [6], [7], [16] and p. 216 in [1]). If $f$ is a smooth function and $f \geq 0$, then

$$
\left(\int_{M} f^{\frac{n}{n-1}} d v\right)^{1-(1 / n)} \leq K(n) \int_{M}(|d f|+|H| f) d v
$$

where $K(n)$ is a constant depending on $n$ and the volume of the unit ball in $\mathbb{R}^{n}$. Taking $f=1$ on $M$, and using the fact that $V(M)=1$, we deduce that

$$
\|H\|_{2 p} \geq \frac{1}{K(n)}
$$

and if $\left(\mathrm{P}_{C}\right)$ is satisfied and $C \leq \frac{n}{2 K(n)^{2}}=c_{n}$, then

$$
\frac{n}{\lambda_{1}(M)} \leq \frac{1}{n\|H\|_{2 p}^{2}-C} \leq 2 K(n)^{2}=d_{n} .
$$

Throughout the paper, we will assume that $V(M)=1$ and $\int_{M} X_{i} d v=0$ for all $i \leq n+1$. The last assertion implies that the center of mass of $M$ is the origin of $\mathbb{R}^{n+1}$.

\section{An $L^{2}$-approach of the problem}

A first step in the proof of Theorem 1.2 is to prove that if the pinching condition $\left(\mathrm{P}_{C}\right)$ is satisfied, then $M$ is close to a sphere in an $L^{2}$-sense.

In the following lemma, we prove that the $L^{2}$-norm of the position vector is close to $\sqrt{\frac{n}{\lambda_{1}(M)}}$. 
Lemma 2.1. If we have the pinching condition $\left(\mathrm{P}_{C}\right)$ with $C<c_{n}$, then

$$
\frac{n \lambda_{1}(M)}{\left(C+\lambda_{1}(M)\right)^{2}} \leq\|X\|_{2}^{2} \leq \frac{n}{\lambda_{1}(M)} \leq d_{n} .
$$

Proof. Since $\int_{M} X_{i} d v=0$, we can apply the variational characterization of the eigenvalues to obtain

$$
\lambda_{1}(M) \int_{M} \sum_{i \leq n+1}\left|X_{i}\right|^{2} d v \leq \int_{M} \sum_{i \leq n+1}\left|d X_{i}\right|^{2} d v=n
$$

which gives the inequality of the right-hand side.

Let us prove now the inequality of the left-hand side.

$$
\begin{aligned}
\lambda_{1}(M) \int_{M}|X|^{2} d v & \leq \frac{\left(\int_{M} \sum_{i \leq n+1}\left|d X_{i}\right|^{2} d v\right)^{4}}{\left(\int_{M} \sum_{i \leq n+1}\left|d X_{i}\right|^{2} d v\right)^{3}}=\frac{\left(\int_{M} \sum_{i \leq n+1}\left(\Delta X_{i}\right) X_{i} d v\right)^{4}}{n^{3}} \\
& \leq \frac{\left(\int_{M} \sum_{i \leq n+1}\left(\Delta X_{i}\right)^{2} d v\right)^{2}\left(\int_{M}|X|^{2} d v\right)^{2}}{n^{3}} \\
& =n\left(\int_{M} H^{2} d v\right)^{2}\left(\int_{M}|X|^{2} d v\right)^{2}
\end{aligned}
$$

then using again the Hölder inequality, we get

$$
\lambda_{1}(M) \leq \frac{1}{n}\left(n\|H\|_{2 p}^{2}\right)^{2} \int_{M}|X|^{2} d v \leq \frac{\left(C+\lambda_{1}(M)\right)^{2}}{n} \int_{M}|X|^{2} d v .
$$

This completes the proof.

From now on, we will denote by $X^{T}$ the orthogonal tangential projection on $M$. In fact, at $x \in M, X^{T}$ is nothing but the vector of $T_{x} M$ defined by $X^{T}=$ $\sum_{1 \leq i \leq n}\left\langle X, e_{i}\right\rangle e_{i}$ where $\left(e_{i}\right)_{1 \leq i \leq n}$ is an orthonormal basis of $T_{x} M$. In the following lemma, we will show that the condition $\left(\mathrm{P}_{C}\right)$ implies that the $L^{2}$-norm of $X^{T}$ of $X$ on $M$ is close to 0 .

Lemma 2.2. If we have the pinching condition $\left(\mathrm{P}_{C}\right)$, then

$$
\left\|X^{T}\right\|_{2}^{2} \leq A(n) C .
$$

Proof. From Lemma 2.1 and the relation (3), we have

$$
\lambda_{1}(M) \int_{M}|X|^{2} d v \leq n=n\left(\int_{M} H\langle X, v\rangle d v\right)^{2}
$$




$$
\begin{aligned}
& \leq\left(\int_{M}|H \|\langle X, v\rangle| d v\right)^{2} \leq n\|H\|_{2 p}^{2}\left(\int_{M}|\langle X, v\rangle|^{\frac{2 p}{2 p-1}} d v\right)^{\frac{2 p-1}{p}} \\
& \leq n\|H\|_{2 p}^{2}\left(\int_{M}|\langle X, v\rangle|^{2} d v\right)=n\|H\|_{2 p}^{2} \int_{M}|X|^{2} d v
\end{aligned}
$$

Then we deduce that

$$
\begin{aligned}
n\|H\|_{2 p}^{2}\left\|X^{T}\right\|_{2}^{2} & =n\|H\|_{2 p}^{2}\left(\int_{M}\left(|X|^{2}-|\langle X, v\rangle|^{2}\right) d v\right) \\
& \leq\left(n\|H\|_{2 p}^{2}-\lambda_{1}(M)\right)\|X\|_{2}^{2} \leq d_{n} C
\end{aligned}
$$

where in the last inequality we have used the pinching condition and Lemma 2.1.

Next we will show that the condition $\left(\mathrm{P}_{C}\right)$ implies that the component functions are almost eigenfunctions in an $L^{2}$-sense. For this, let us consider the vector field $Y$ on $M$ defined by

$$
Y=\sum_{i \leq n+1}\left(\Delta X_{i}-\lambda_{1}(M) X_{i}\right) \partial_{i}=n H v-\lambda_{1}(M) X .
$$

Lemma 2.3. If $\left(\mathrm{P}_{C}\right)$ is satisfied, then

$$
\|Y\|_{2}^{2} \leq n C .
$$

Proof. We have

$$
\int_{M}|Y|^{2} d v=\int_{M}\left(n^{2} H^{2}-2 n \lambda_{1}(M) H\langle v, X\rangle+\lambda_{1}(M)^{2}|X|^{2}\right) d v .
$$

Now by integrating the relation (3) we deduce that

$$
\int_{M} H\langle v, X\rangle d v=1
$$

Furthermore, since $\int_{M} X_{i} d v=0$, we can apply the variational characterization of the eigenvalues to obtain

$$
\lambda_{1}(M) \int_{M}|X|^{2} d v=\lambda_{1}(M) \int_{M} \sum_{i \leq n+1}\left|X_{i}\right|^{2} d v \leq \int_{M} \sum_{i \leq n+1}\left|d X_{i}\right|^{2} d v=n .
$$

Then

$$
\int_{M}|Y|^{2} d v \leq n^{2} \int_{M}|H|^{2} d v-n \lambda_{1}(M) \leq n\left(n\|H\|_{2 p}^{2}-\lambda_{1}(M)\right) \leq n C
$$

where in this last inequality we have used the Hölder inequality. 
To prove Assertion 1 of Theorem 1.2, we will show that $\left\||X|-\left(\frac{n}{\lambda_{1}(M)}\right)^{1 / 2}\right\|_{\infty} \leq \varepsilon$. For this we need an $L^{2}$-upper bound on the function $\varphi=|X|\left(|X|-\left(\frac{n}{\lambda_{1}(M)}\right)^{1 / 2}\right)^{2}$.

Before giving such estimate, we will introduce the vector field $Z$ on $M$ defined by

$$
Z=\left(\frac{n}{\lambda_{1}(M)}\right)^{1 / 2}|X|^{1 / 2} H v-\frac{X}{|X|^{1 / 2}}
$$

We have

Lemma 2.4. If $\left(\mathrm{P}_{C}\right)$ is satisfied with $C<c_{n}$, then

$$
\|Z\|_{2}^{2} \leq B(n) C \text {. }
$$

Proof. We have

$$
\begin{aligned}
\|Z\|_{2}^{2}= & \left\|\left(\frac{n}{\lambda_{1}(M)}\right)^{1 / 2}|X|^{1 / 2} H v-\frac{X}{|X|^{1 / 2}}\right\|_{2}^{2} \\
= & \int_{M}\left(\frac{n}{\lambda_{1}(M)}|X| H^{2}-2\left(\frac{n}{\lambda_{1}(M)}\right)^{1 / 2} H\langle v, X\rangle+|X|\right) d v \\
\leq & \frac{n}{\lambda_{1}(M)}\left(\int_{M}|X|^{2} d v\right)^{1 / 2}\left(\int_{M} H^{4} d v\right)^{1 / 2} \\
& -2\left(\frac{n}{\lambda_{1}(M)}\right)^{1 / 2}+\left(\int_{M}|X|^{2} d v\right)^{1 / 2} .
\end{aligned}
$$

Note that we have used the relation (3). Finally for $p \geq 2$, we get

$$
\begin{aligned}
\|Z\|_{2}^{2} & \leq\left(\int_{M}|X|^{2} d v\right)^{1 / 2}\left(\frac{n}{\lambda_{1}(M)}\|H\|_{2 p}^{2}+1\right)-2\left(\frac{n}{\lambda_{1}(M)}\right)^{1 / 2} \\
& \leq\left(\frac{n}{\lambda_{1}(M)}\right)^{1 / 2}\left(\frac{C}{\lambda_{1}(M)}+2\right)-2\left(\frac{n}{\lambda_{1}(M)}\right)^{1 / 2} \\
& =\left(\frac{n}{\lambda_{1}(M)}\right)^{1 / 2} \frac{C}{\lambda_{1}(M)} \leq \frac{d_{n}^{3 / 2}}{n} C .
\end{aligned}
$$

This concludes the proof of the lemma.

Now we give an $L^{2}$-upper bound of $\varphi$.

Lemma 2.5. Let $p \geq 2$ and $C \leq c_{n}$. If we have the pinching condition $\left(\mathrm{P}_{C}\right)$, then

$$
\|\varphi\|_{2} \leq D(n)\|\varphi\|_{\infty}^{3 / 4} C^{1 / 4} .
$$


Proof. We have

$$
\|\varphi\|_{2}=\left(\int_{M} \varphi^{3 / 2} \varphi^{1 / 2} d v\right)^{1 / 2} \leq\|\varphi\|_{\infty}^{3 / 4}\left\|\varphi^{1 / 2}\right\|_{1}^{1 / 2},
$$

and noting that

$$
|X|\left(|X|-\left(\frac{n}{\lambda_{1}(M)}\right)^{1 / 2}\right)^{2}=\left.|| X\right|^{1 / 2} X-\left.\left(\frac{n}{\lambda_{1}(M)}\right)^{1 / 2} \frac{X}{|X|^{1 / 2}}\right|^{2}
$$

we get

$$
\begin{aligned}
\int_{M} \varphi^{1 / 2} d v & =\left\||X|^{1 / 2} X-\left(\frac{n}{\lambda_{1}(M)}\right)^{1 / 2} \frac{X}{|X|^{1 / 2}}\right\|_{1} \\
& =\left\|-\frac{|X|^{1 / 2}}{\lambda_{1}(M)} Y+\frac{n}{\lambda_{1}(M)}|X|^{1 / 2} H v-\left(\frac{n}{\lambda_{1}(M)}\right)^{1 / 2} \frac{X}{|X|^{1 / 2}}\right\|_{1} \\
& \leq\left\|\frac{|X|^{1 / 2}}{\lambda_{1}(M)} Y\right\|_{1}+\left(\frac{n}{\lambda_{1}(M)}\right)^{1 / 2}\|Z\|_{1} .
\end{aligned}
$$

From Lemmas 2.3 and 1.1 we get

$$
\begin{aligned}
\left\|\frac{|X|^{1 / 2}}{\lambda_{1}(M)} Y\right\|_{1} & \leq \frac{1}{\lambda_{1}(M)}\left(\int_{M}|X| d v\right)^{1 / 2}\|Y\|_{2} \\
& \leq \frac{1}{\lambda_{1}(M)}\left(\int_{M}|X|^{2} d v\right)^{1 / 4}\|Y\|_{2} \leq \frac{d_{n}^{3 / 4}}{n^{1 / 2}} C^{1 / 2} .
\end{aligned}
$$

Moreover, using Lemmas 2.4 and 1.1 again it is easy to see that the last term of (6) is bounded by $d_{n}^{1 / 2} B(n)^{1 / 2} C^{1 / 2}$. Then $\left\|\varphi^{1 / 2}\right\|_{1}^{1 / 2} \leq D(n) C^{1 / 4}$.

\section{Proof of Theorem 1.2}

The proof of Theorem 1.2 is immediate from the two following technical lemmas which we state below.

Lemma 3.1. For $p \geq 2$ and for any $\eta>0$, there exists $K_{\eta}\left(n,\|H\|_{\infty}\right) \leq c_{n}$ so that if $\left(\mathrm{P}_{K_{\eta}}\right)$ is true, then $\|\varphi\|_{\infty} \leq \eta$. Moreover, $K_{\eta} \rightarrow 0$ when $\|H\|_{\infty} \rightarrow \infty$ or $\eta \rightarrow 0$.

Lemma 3.2. Let $x_{0}$ be a point of the sphere $S(O, R)$ of $\mathbb{R}^{n+1}$ with the center at the origin and of radius $R$. Assume that $x_{0}=R e$ where $e \in \mathbb{S}^{n}$. Now let $\left(M^{n}, g\right)$ be a compact oriented n-dimensional Riemannian manifold without boundary isometrically 
immersed by $\phi$ in $\mathbb{R}^{n+1}$ so that $\phi(M) \subset(B(O, R+\eta) \backslash B(O, R-\eta)) \backslash B\left(x_{0}, \rho\right)$ with $\rho=4(2 n-1) \eta$ and suppose that there exists a point $p \in M$ so that $\langle X, e\rangle>0$. Then there exists $y_{0} \in M$ so that the mean curvature $H\left(y_{0}\right)$ at $y_{0}$ satisfies $\left|H\left(y_{0}\right)\right| \geq \frac{1}{4 n \eta}$.

Now, let us see how to use these lemmas to prove Theorem 1.2.

Proof of Theorem 1.2. We consider the function $f(t)=t\left(t-\left(\frac{n}{\lambda_{1}(M)}\right)^{1 / 2}\right)^{2}$. For $\varepsilon>0$ let us put

$$
\begin{aligned}
\eta(\varepsilon) & =\min \left(\left(\frac{1}{\|H\|_{\infty}}-\varepsilon\right) \varepsilon^{2},\left(\frac{1}{\|H\|_{\infty}}+\varepsilon\right) \varepsilon^{2}, \frac{1}{27\|H\|_{\infty}^{3}}\right) \\
& \leq \min \left(f\left(\left(\frac{n}{\lambda_{1}(M)}\right)^{1 / 2}-\varepsilon\right), f\left(\left(\frac{n}{\lambda_{1}(M)}\right)^{1 / 2}+\varepsilon\right), \frac{1}{27\|H\|_{\infty}^{3}}\right) .
\end{aligned}
$$

Then, as $\eta(\varepsilon)>0$ and from Lemma 3.1, it follows that if the pinching condition $\left(\mathrm{P}_{K_{\eta(\varepsilon)}}\right)$ is satisfied with $K_{\eta(\varepsilon)} \leq c_{n}$, then for any $x \in M$, we have

$$
f(|X|) \leq \eta(\varepsilon)
$$

Now to prove Theorem 1.2, it is sufficient to assume $\varepsilon<\frac{2}{3\|H\|_{\infty}}$. Let us show that either

$$
\left(\frac{n}{\lambda_{1}(M)}\right)^{1 / 2}-\varepsilon \leq|X| \leq\left(\frac{n}{\lambda_{1}(M)}\right)^{1 / 2}+\varepsilon \quad \text { or } \quad|X|<\frac{1}{3}\left(\frac{n}{\lambda_{1}(M)}\right)^{1 / 2} .
$$

By studying the function $f$ it is easy to see that $f$ has a unique local maximum in $\frac{1}{3}\left(\frac{n}{\lambda_{1}(M)}\right)^{1 / 2}$ and from the definition of $\eta(\varepsilon)$ it follows that $\eta(\varepsilon)<\frac{4}{27} \frac{1}{\|H\|_{\infty}^{3}} \leq$ $\frac{4}{27}\left(\frac{n}{\lambda_{1}(M)}\right)^{3 / 2}=f\left(\frac{1}{3}\left(\frac{n}{\lambda_{1}(M)}\right)^{1 / 2}\right)$.

Since $\varepsilon<\frac{2}{3\|H\|_{\infty}}$, we have $\varepsilon<\frac{2}{3}\left(\frac{n}{\lambda_{1}(M)}\right)^{1 / 2}$ and $\frac{1}{3}\left(\frac{n}{\lambda_{1}(M)}\right)^{1 / 2}<\left(\frac{n}{\lambda_{1}(M)}\right)^{1 / 2}-\varepsilon$. This and (7) yield (8).

Now, from Lemma 2.1 we deduce that there exists a point $y_{0} \in M$ so that $\left|X\left(y_{0}\right)\right| \geq \frac{n^{1 / 2} \lambda_{1}(M)^{1 / 2}}{\left(K_{\eta(\varepsilon)}+\lambda_{1}(M)\right)}$ and since $K_{\eta(\varepsilon)} \leq c_{n}=\frac{n}{d_{n}} \leq \lambda_{1}(M) \leq 2 \lambda_{1}(M)$ (see the proof of Lemma 1.1), we obtain $\left|X\left(y_{0}\right)\right| \geq \frac{1}{3}\left(\frac{n}{\lambda_{1}(M)}\right)^{1 / 2}$.

By the connectedness of $M$, it follows that $\left(\frac{n}{\lambda_{1}(M)}\right)^{1 / 2}-\varepsilon \leq|X| \leq\left(\frac{n}{\lambda_{1}(M)}\right)^{1 / 2}+\varepsilon$ for any point of $M$ and Assertion 1 of Theorem 1.2 is shown for the condition $\left(\mathrm{P}_{K_{\eta(\varepsilon)}}\right)$.

In order to prove the second assertion, let us consider the pinching condition $\left(\mathrm{P}_{C_{\varepsilon}}\right)$ with $C_{\varepsilon}=K_{\eta\left(\frac{\varepsilon}{4(2 n-1)}\right)}$. Then Assertion 1 is still valid. Let $x=\left(\frac{n}{\lambda_{1}(M)}\right)^{1 / 2} e \in$ $S\left(O, \sqrt{\frac{n}{\lambda_{1}(M)}}\right)$, with $e \in \mathbb{S}^{n}$ and suppose that $B(x, \varepsilon) \cap M=\emptyset$. Since $\int_{M} X_{i} d v=0$ 
for any $i \leq n+1$, there exists a point $p \in M$ so that $\langle X, e\rangle>0$ and we can apply Lemma 3.2. Therefore there is a point $y_{0} \in M$ so that $H\left(y_{0}\right) \geq \frac{2 n-1}{n \varepsilon}>\|H\|_{\infty}$ since we have assumed $\varepsilon<\frac{2}{3\|H\|_{\infty}} \leq \frac{2 n-1}{2 n\|H\|_{\infty}}$. Then we obtain a contradiction which implies $B(x, \varepsilon) \cap M \neq \varnothing$ and Assertion 2 is satisfied. Furthermore, $C_{\varepsilon} \rightarrow 0$ when $\|H\|_{\infty} \rightarrow \infty$ or $\varepsilon \rightarrow 0$.

\section{Proof of Theorem 1.3}

From Theorem 1.2, we know that for any $\varepsilon>0$, there exists $C_{\varepsilon}$ depending only on $n$ and $\|H\|_{\infty}$ so that if $\left(\mathrm{P}_{C_{\varepsilon}}\right)$ is true then

$$
\left.|| X\right|_{x}-\sqrt{\frac{n}{\lambda_{1}(M)}} \mid \leq \varepsilon
$$

for any $x \in M$. Now, since $\sqrt{n}\|H\|_{\infty} \leq\|B\|_{\infty}$, it is easy to see from the previous proofs that we can assume that $C_{\varepsilon}$ is depending only on $n$ and $\|B\|_{\infty}$.

The proof of Theorem 1.3 is a consequence of the following lemma on the $L_{\infty^{-}}$norm of $\psi=\left|X^{T}\right|$.

Lemma 4.1. For $p \geq 2$ and for any $\eta>0$, there exists $K_{\eta}\left(n,\|B\|_{\infty}\right)$ so that if $\left(\mathrm{P}_{K_{\eta}}\right)$ is true, then $\|\psi\|_{\infty} \leq \eta$. Moreover, $K_{\eta} \rightarrow 0$ when $\|B\|_{\infty} \rightarrow \infty$ or $\eta \rightarrow 0$.

This lemma will be proved in the Section 5.

Proof of Theorem 1.3. Let $\varepsilon<\frac{1}{2} \sqrt{\frac{n}{\|B\|_{\infty}}} \leq \sqrt{\frac{n}{\lambda_{1}(M)}}$. From the choice of $\varepsilon$, we deduce that the condition $\left(\mathrm{P}_{C_{\varepsilon}}\right)$ implies that $\left|X_{x}\right|$ is nonzero for any $x \in M$ (see the proof of Theorem 1.2) and we can consider the differential application

$$
\begin{aligned}
F: M & \longrightarrow S\left(O, \sqrt{\frac{n}{\lambda_{1}(M)}}\right), \\
x & \longmapsto \sqrt{\frac{n}{\lambda_{1}(M)} \frac{X_{x}}{\left|X_{x}\right|} .}
\end{aligned}
$$

We will prove that $F$ is a quasi-isometry. Indeed, for any $0<\theta<1$, we can choose a constant $\varepsilon\left(n,\|B\|_{\infty}, \theta\right)$ so that for any $x \in M$ and any unit vector $u \in T_{x} M$, the pinching condition $\left(\mathrm{P}_{C_{\varepsilon(n,\|B\| \infty, \theta)}}\right)$ implies

$$
\left.|| d F_{x}(u)\right|^{2}-1 \mid \leq \theta .
$$

For this, let us compute $d F_{x}(u)$. We have

$$
d F_{x}(u)=\left.\sqrt{\frac{n}{\lambda_{1}(M)}} \nabla_{u}^{0}\left(\frac{X}{|X|}\right)\right|_{x}=\sqrt{\frac{n}{\lambda_{1}(M)}} u\left(\frac{1}{|X|}\right) X+\sqrt{\frac{n}{\lambda_{1}(M)}} \frac{1}{|X|} \nabla_{u}^{0} X=
$$




$$
\begin{aligned}
& =-\frac{1}{2} \sqrt{\frac{n}{\lambda_{1}(M)}} \frac{1}{|X|^{3}} u\left(|X|^{2}\right) X+\sqrt{\frac{n}{\lambda_{1}(M)}} \frac{1}{|X|} u \\
& =-\sqrt{\frac{n}{\lambda_{1}(M)}} \frac{1}{|X|^{3}}\langle u, X\rangle X+\sqrt{\frac{n}{\lambda_{1}(M)}} \frac{1}{|X|} u \\
& =\sqrt{\frac{n}{\lambda_{1}(M)}} \frac{1}{|X|}\left(-\frac{\langle u, X\rangle}{|X|^{2}} X+u\right) .
\end{aligned}
$$

By a straightforward computation, we obtain

$$
\begin{aligned}
\left.|| d F_{x}(u)\right|^{2}-1 \mid & =\left|\frac{n}{\lambda_{1}(M)} \frac{1}{|X|^{2}}\left(1-\frac{\langle u, X\rangle^{2}}{|X|^{2}}\right)-1\right| \\
& \leq\left|\frac{n}{\lambda_{1}(M)} \frac{1}{|X|^{2}}-1\right|+\frac{n}{\lambda_{1}(M)} \frac{1}{|X|^{4}}\langle u, X\rangle^{2} .
\end{aligned}
$$

Now

$$
\begin{aligned}
\left|\frac{n}{\lambda_{1}(M)} \frac{1}{|X|^{2}}-1\right| & =\left.\frac{1}{|X|^{2}}\left|\frac{n}{\lambda_{1}(M)}-\right| X\right|^{2} \mid \\
& \leq \varepsilon \frac{\left|\sqrt{\frac{n}{\lambda_{1}(M)}}+\right| X||}{|X|^{2}} \leq \varepsilon \frac{2 \sqrt{\frac{n}{\lambda_{1}(M)}}+\varepsilon}{\left(\sqrt{\frac{n}{\lambda_{1}(M)}}-\varepsilon\right)^{2}} .
\end{aligned}
$$

Let us recall that $\frac{n}{d_{n}} \leq \lambda_{1}(M) \leq\|B\|_{\infty}^{2}$ (see (4) for the first inequality). Since we assume $\varepsilon<\frac{1}{2} \sqrt{\frac{n}{\|B\|_{\infty}}}$, the right-hand side is bounded above by a constant depending only on $n$ and $\|B\|_{\infty}$ and we have

$$
\left|\frac{n}{\lambda_{1}(M)} \frac{1}{|X|^{2}}-1\right| \leq \varepsilon \gamma\left(n,\|B\|_{\infty}\right) .
$$

On the other hand, since $C_{\varepsilon}\left(n,\|B\|_{\infty}\right) \rightarrow 0$ when $\varepsilon \rightarrow 0$, there exists $\varepsilon\left(n,\|B\|_{\infty}, \eta\right)$

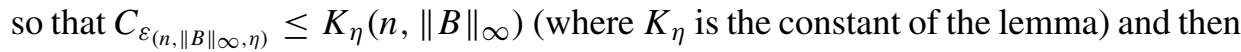
by Lemma $4.1,\|\psi\|_{\infty}^{2} \leq \eta^{2}$. Thus there exists a constant $\delta$ depending only on $n$ and $\|B\|_{\infty}$ so that

$$
\frac{n}{\lambda_{1}(M)} \frac{1}{|X|^{4}}\langle u, X\rangle^{2} \leq \frac{n}{\lambda_{1}(M)} \frac{1}{|X|^{4}}\|\psi\|_{\infty}^{2} \leq \eta^{2} \delta\left(n,\|B\|_{\infty}\right),
$$

and from (9), (10) and (11) we deduce that the condition $\left(\mathrm{P}_{C_{\varepsilon(n,\|B\| \infty, \eta)}}\right)$ implies

$$
\left.|| d F_{x}(u)\right|^{2}-1 \mid \leq \varepsilon \gamma\left(n,\|B\|_{\infty}\right)+\eta^{2} \delta\left(n,\|B\|_{\infty}\right) .
$$


Now let us choose $\eta=\left(\frac{\theta}{2 \delta}\right)^{1 / 2}$. Then we can assume that $\varepsilon\left(n,\|B\|_{\infty}, \eta\right)$ is small enough in order to have $\varepsilon\left(n,\|B\|_{\infty}, \eta\right) \gamma\left(n\|B\|_{\infty}\right) \leq \frac{\theta}{2}$. In this case we have

$$
\left.|| d F_{x}(u)\right|^{2}-1 \mid \leq \theta \text {. }
$$

Now let us fix $\theta, 0<\theta<1$. It follows that $F$ is a local diffeomorphism from $M$ to $S\left(O, \sqrt{\frac{n}{\lambda_{1}(M)}}\right)$. Since $S\left(O, \sqrt{\frac{n}{\lambda_{1}(M)}}\right)$ is simply connected for $n \geq 2, F$ is a diffeomorphism.

\section{Proof of the technical lemmas}

The proofs of Lemmas 3.1 and 4.1 are providing from a result stated in the following proposition using a Nirenberg-Moser type of proof.

Proposition 5.1. Let $\left(M^{n}, g\right)$ be a compact, connected and oriented $n$-dimensional Riemannian manifold without boundary isometrically immersed into the $n+1$-dimensional euclidean space $\left(\mathbb{R}^{n+1}\right.$, can). Let $\xi$ be a nonnegative continuous function so that $\xi^{k}$ is smooth for $k \geq 2$. Let $0 \leq r<s \leq 2$ so that

$$
\frac{1}{2} \Delta \xi^{2} \xi^{2 k-2} \leq \delta \omega+\left(A_{1}+k A_{2}\right) \xi^{2 k-r}+\left(B_{1}+k B_{2}\right) \xi^{2 k-s}
$$

where $\delta \omega$ is the codifferential of a 1-form and $A_{1}, A_{2}, B_{1}, B_{2}$ are nonnegative constants. Then for any $\eta>0$, there exists a constant $L\left(n, A_{1}, A_{2}, B_{1}, B_{2},\|H\|_{\infty}, \eta\right)$ depending only on $n, A_{1}, A_{2}, B_{1}, B_{2},\|H\|_{\infty}$ and $\eta$ so that if $\|\xi\|_{\infty}>\eta$ then

$$
\|\xi\|_{\infty} \leq L\left(n, A_{1}, A_{2}, B_{1}, B_{2},\|H\|_{\infty}, \eta\right)\|\xi\|_{2} .
$$

Moreover, $L$ is bounded when $\eta \rightarrow \infty$, and if $B_{1}>0, L \rightarrow \infty$ when $\|H\|_{\infty} \rightarrow \infty$ or $\eta \rightarrow 0$.

This proposition will be proved at the end of the paper.

Before giving the proofs of Lemmas 3.1 and 4.1, we will show that under the pinching condition $\left(\mathrm{P}_{C}\right)$ with $C$ small enough, the $L_{\infty}$-norm of $X$ is bounded by a constant depending only on $n$ and $\|H\|_{\infty}$.

Lemma 5.1. If we have the pinching condition $\left(\mathrm{P}_{C}\right)$ with $C<c_{n}$, then there exists $E\left(n,\|H\|_{\infty}\right)$ depending only on $n$ and $\|H\|_{\infty}$ so that $\|X\|_{\infty} \leq E\left(n,\|H\|_{\infty}\right)$.

Proof. From the relation (3), we have

$$
\frac{1}{2} \Delta|X|^{2}|X|^{2 k-2} \leq n\|H\|_{\infty}|X|^{2 k-1} .
$$


Then applying Proposition 5.1 to the function $\xi=|X|$ with $r=0$ and $s=1$, we obtain that if $\|X\|_{\infty}>E$, then there exists a constant $L\left(n,\|H\|_{\infty}, E\right)$ depending only on $n,\|H\|_{\infty}$ and $E$ so that

$$
\|X\|_{\infty} \leq L\left(n,\|H\|_{\infty}, E\right)\|X\|_{2},
$$

and under the pinching condition $\left(\mathrm{P}_{C}\right)$ with $C<c_{n}$ we have from Lemma 2.1 that

$$
\|X\|_{\infty} \leq L\left(n,\|H\|_{\infty}, E\right) d_{n}^{1 / 2} .
$$

Now since $L$ is bounded when $E \rightarrow \infty$, we can choose $E=E\left(n,\|H\|_{\infty}\right)$ large enough so that

$$
L\left(n,\|H\|_{\infty}, E\right) d_{n}^{1 / 2}<E .
$$

In this case, we have $\|X\|_{\infty} \leq E\left(n,\|H\|_{\infty}\right)$.

Proof of Lemma 3.1. First we compute the Laplacian of the square of $\varphi^{2}$. We have

$$
\begin{aligned}
\Delta \varphi^{2} & =\Delta\left(|X|^{4}-2\left(\frac{n}{\lambda_{1}(M)}\right)^{1 / 2}|X|^{3}+\frac{n}{\lambda_{1}(M)}|X|^{2}\right) \\
& =-\left.\left.2|X|^{2}|d| X\right|^{2}\right|^{2}+2|X|^{2} \Delta|X|^{2} \\
& -2\left(\frac{n}{\lambda_{1}(M)}\right)^{1 / 2}\left(-\left.\left.\frac{3}{4}|X|^{-1}|d| X\right|^{2}\right|^{2}+\frac{3}{2}|X| \Delta|X|^{2}\right)+\frac{n}{\lambda_{1}(M)} \Delta|X|^{2} .
\end{aligned}
$$

Now by a direct computation one gets $\left.\left.|d| X\right|^{2}\right|^{2} \leq 4|X|^{2}$. Moreover by the relation (3) we have $\left.|\Delta| X\right|^{2}\left|\leq 2 n\|H\|_{\infty}\right| X \mid+n$. Then applying Lemmas 1.1 and 5.1 we get

$$
\Delta \varphi^{2} \leq \alpha\left(n,\|H\|_{\infty}\right)
$$

and

$$
\frac{1}{2} \Delta \varphi^{2} \varphi^{2 k-2} \leq \alpha\left(n,\|H\|_{\infty}\right) \varphi^{2 k-2} .
$$

Now, we apply Proposition 5.1 with $r=0$ and $s=2$. Then if $\|\varphi\|_{\infty}>\eta$, there exists a constant $L\left(n,\|H\|_{\infty}\right)$ depending only on $n$ and $\|H\|_{\infty}$ so that

$$
\|\varphi\|_{\infty} \leq L\|\varphi\|_{2}
$$

From Lemma 2.5, if $C \leq c_{n}$ and $\left(\mathrm{P}_{C}\right)$ is true, we have $\|\varphi\|_{2} \leq D(n)\|\varphi\|_{\infty}^{3 / 4} C^{1 / 4}$. Therefore

$$
\|\varphi\|_{\infty} \leq(L D)^{4} C .
$$

Consequently, if we choose $C=K_{\eta}=\inf \left(\frac{\eta}{(L D)^{4}}, c_{n}\right)$, then we obtain $\|\varphi\|_{\infty} \leq \eta$. 
Proof of Lemma 4.1. First we will prove that for any $C<c_{n}$, if $\left(\mathrm{P}_{C}\right)$ is true, then

$$
\frac{1}{2}\left(\Delta \psi^{2}\right) \psi^{2 k-2} \leq \delta \omega+\left(\alpha_{1}\left(n,\|B\|_{\infty}\right)+k \alpha_{2}\left(n,\|B\|_{\infty}\right)\right) \psi^{2 k-2}
$$

where $\delta \omega$ is the codifferential of a 1-form $\omega$.

First observe that the gradient $\nabla^{M}|X|^{2}$ of $|X|^{2}$ satisfies $\nabla^{M}|X|^{2}=2 X^{T}$. Then by the Bochner formula we get

$$
\begin{aligned}
\frac{1}{2} \Delta\left|X^{T}\right|^{2} & =\frac{1}{4}\left\langle\Delta d|X|^{2}, d|X|^{2}\right\rangle-\left.\left.\frac{1}{4}|\nabla d| X\right|^{2}\right|^{2}-\frac{1}{4} \operatorname{Ric}\left(\nabla^{M}|X|^{2}, \nabla^{M}|X|^{2}\right) \\
& \leq \frac{1}{4}\left\langle d \Delta|X|^{2}, d|X|^{2}\right\rangle-\frac{1}{4} \operatorname{Ric}\left(\nabla^{M}|X|^{2}, \nabla^{M}|X|^{2}\right)
\end{aligned}
$$

and by the Gauss formula we obtain

$$
\begin{aligned}
\frac{1}{2} \Delta\left|X^{T}\right|^{2} & \leq \frac{1}{4}\left\langle d \Delta|X|^{2}, d|X|^{2}\right\rangle-\frac{1}{4} n H\left\langle B \nabla^{M}|X|^{2}, \nabla^{M}|X|^{2}\right\rangle+\left.\left.\frac{1}{4}\left|B \nabla^{M}\right| X\right|^{2}\right|^{2} \\
& =\frac{1}{4}\left\langle d \Delta|X|^{2}, d|X|^{2}\right\rangle-n H\left\langle B X^{T}, X^{T}\right\rangle+\left|B X^{T}\right|^{2} .
\end{aligned}
$$

By Lemma 5.1 we know that $\|X\|_{\infty} \leq E\left(n,\|B\|_{\infty}\right)$ (the dependance in $\|H\|_{\infty}$ can be replaced by $\left.\|B\|_{\infty}\right)$. Then it follows that

$$
\frac{1}{2}\left(\Delta \psi^{2}\right) \psi^{2 k-2} \leq \frac{1}{4}\left\langle d \Delta|X|^{2}, d|X|^{2}\right\rangle \psi^{2 k-2}+\alpha^{\prime}\left(n,\|B\|_{\infty}\right) \psi^{2 k-2} .
$$

Now, let us compute the term $\left\langle d \Delta|X|^{2}, d|X|^{2}\right\rangle \psi^{2 k-2}$. We have

$$
\begin{aligned}
\left\langle d \Delta|X|^{2},\right. & \left.d|X|^{2}\right\rangle \psi^{2 k-2} \\
= & \delta \omega+\left(\Delta|X|^{2}\right)^{2} \psi^{2 k-2}-(2 k-2) \Delta|X|^{2}\left\langle d|X|^{2}, d \psi\right\rangle \psi^{2 k-3} \\
& =\delta \omega+\left(\Delta|X|^{2}\right)^{2} \psi^{2 k-2}-2(2 k-2) \Delta|X|^{2}\left\langle X^{T}, \nabla^{M} \psi\right\rangle \psi^{2 k-3}
\end{aligned}
$$

where $\omega=-\Delta|X|^{2} \psi^{2 k-2} d|X|^{2}$. Now,

$$
e_{i}(\psi)=\frac{e_{i}\left|X^{T}\right|^{2}}{2\left|X^{T}\right|}=\frac{e_{i}|X|^{2}-e_{i}\langle X, v\rangle^{2}}{2\left|X^{T}\right|}=\frac{\left\langle e_{i}, X\right\rangle-B_{i j}\left\langle X, e_{j}\right\rangle\langle X, v\rangle}{\left|X^{T}\right|} .
$$

Then

$$
\begin{aligned}
& \left\langle d \Delta|X|^{2}, d|X|^{2}\right\rangle \psi^{2 k-2}=\delta \omega+\left(\Delta|X|^{2}\right)^{2} \psi^{2 k-2}-2(2 k-2) \Delta|X|^{2}\left|X^{T}\right| \psi^{2 k-3} \\
& +2(2 k-2) \Delta|X|^{2} \frac{\left\langle B X^{T}, X^{T}\right\rangle}{\left|X^{T}\right|}\langle X, v\rangle \psi^{2 k-3} \\
& \leq \delta \omega+\left(\Delta|X|^{2}\right)^{2} \psi^{2 k-2}+\left.2(2 k-2)|\Delta| X\right|^{2} \mid \psi^{2 k-2} \\
& +\left.2(2 k-2)|\Delta| X\right|^{2}|| B|| X \mid \psi^{2 k-2} \text {. }
\end{aligned}
$$


Now by relation (3) and Lemma 5.1 we have

$$
\left\langle d \Delta|X|^{2}, d|X|^{2}\right\rangle \psi^{2 k-2} \leq \delta \omega+\left(\alpha_{1}^{\prime \prime}\left(n,\|B\|_{\infty}\right)+k \alpha_{2}^{\prime \prime}\left(n,\|B\|_{\infty}\right)\right) \psi^{2 k-2} .
$$

Inserting this in (13), we obtain the desired inequality (12).

Now applying again Proposition 5.1, we get that there exists $L\left(n,\|B\|_{\infty}, \eta\right)$ so that if $\|\psi\|_{\infty}>\eta$ then

$$
\|\psi\|_{\infty} \leq L\|\psi\|_{2}
$$

From Lemma 2.2 we deduce that if the pinching condition $\left(\mathrm{P}_{C}\right)$ holds then $\|\psi\|_{2} \leq$ $A(n)^{1 / 2} C^{1 / 2}$. Then taking $C=K_{\eta}=\inf \left(\frac{\eta}{L A^{1 / 2}}, c_{n}\right)$, then $\|\psi\|_{\infty} \leq \eta$.

Proof of Lemma 3.2. The idea of the proof consists in foliating the region $B(O, R+\eta) \backslash B(O, R-\eta)$ with hypersurfaces of large mean curvature and to show that one of these hypersurfaces is tangent to $\phi(M)$. This will imply that $\phi(M)$ has a large mean curvature at the contact point.

Consider $\mathbb{S}^{n-1} \subset \mathbb{R}^{n}$ and $\mathbb{R}^{n+1}=\mathbb{R}^{n} \times \mathbb{R} e$. Let $a, L>l>0$ and

$$
\begin{aligned}
\Phi_{L, l, a}: \mathbb{S}^{n-1} \times \mathbb{S}^{1} & \longrightarrow \mathbb{R}^{n+1} \\
(\xi, \theta) & \longmapsto L \xi-l \cos \theta \xi+l \sin \theta e+a e .
\end{aligned}
$$

Then $\Phi_{L, l, a}$ is a family of embeddings from $\mathbb{S}^{n-1} \times \mathbb{S}^{1}$ in $\mathbb{R}^{n+1}$. If we orient the family of hypersurfaces $\Phi_{L, l, a}\left(\mathbb{S}^{n-1} \times \mathbb{S}^{1}\right)$ by the unit outward normal vector field, a straightforward computation shows that the mean curvature $H(\theta)$ depends only on $\theta$ and we have

$$
H(\theta)=\frac{1}{n}\left(\frac{1}{l}-\frac{(n-1) \cos \theta}{L-l \cos \theta}\right) \geq \frac{1}{n}\left(\frac{1}{l}-\frac{n-1}{L-l}\right) .
$$

Now, let us consider the hypotheses of the lemma and for $t_{0}=2 \arcsin \left(\frac{\rho}{2 R}\right) \leq$ $t \leq \frac{\pi}{2}$, put $L=R \sin t, l=2 \eta$ and $a=R \cos t$. Then $L>l$ and we can consider for $t_{0} \leq t \leq \frac{\pi}{2}$ the family $\mathcal{M}_{R, \eta, t}$ of hypersurfaces defined by $\mathcal{M}_{R, \eta, t}=$ $\Phi_{R \sin t, 2 \eta, R \cos t}\left(\mathbb{S}^{n-1} \times \mathbb{S}^{1}\right)$.

From the relation (14), the mean curvature $H_{R, \eta, t}$ of $\mathcal{M}_{R, \eta, t}$ satisfies

$$
\begin{aligned}
H_{R, \eta, t} & \geq \frac{1}{n}\left(\frac{1}{2 \eta}-\frac{n-1}{R \sin t-2 \eta}\right) \geq \frac{1}{n}\left(\frac{1}{2 \eta}-\frac{n-1}{R \sin t_{0}-2 \eta}\right) \\
& \geq \frac{1}{n}\left(\frac{1}{2 \eta}-\frac{n-1}{R \sin \left(t_{0} / 2\right)-2 \eta}\right)=\frac{1}{n}\left(\frac{1}{2 \eta}-\frac{n-1}{\frac{\rho}{2}-2 \eta}\right)=\frac{1}{4 n \eta}
\end{aligned}
$$

where we have used in this last equality the fact that $\rho=4(2 n-1) \eta$.

Since there exists a point $p \in M$ so that $\langle X(p), e\rangle>0$, we can find $t \in\left[t_{0}, \pi / 2\right]$ and a point $y_{0} \in M$ which is a contact point with $\mathcal{M}_{R, \eta, t}$. Therefore $\left|H\left(y_{0}\right)\right| \geq \frac{1}{4 n \eta}$. 


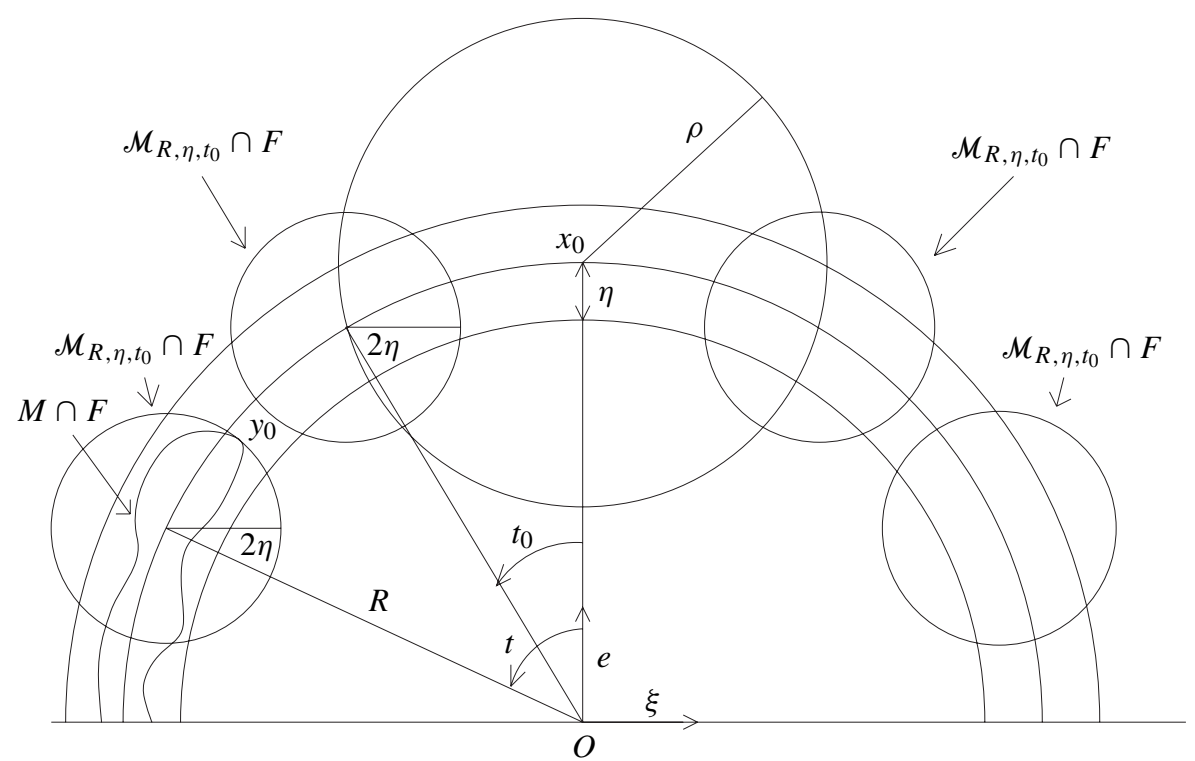

$F$ is the vector space spanned by $e$ and $\xi$.

Proof of Proposition 5.1. Integrating by parts we have

$$
\begin{aligned}
\int_{M} \frac{1}{2} \Delta \xi^{2} \xi^{2 k-2} d v & =\frac{1}{2} \int_{M}\left\langle d \xi^{2}, d \xi^{2 k-2}\right\rangle d v=2\left(\frac{k-1}{k^{2}}\right) \int_{M}\left|d \xi^{k}\right|^{2} d v \\
& \leq\left(A_{1}+k A_{2}\right) \int_{M} \xi^{2 k-r} d v+\left(B_{1}+k B_{2}\right) \int_{M} \xi^{2 k-s} d v
\end{aligned}
$$

Now, given a smooth function $f$ and applying the Sobolev inequality (5) to $f^{2}$, we get

$$
\begin{aligned}
& \left(\int_{M} f^{\frac{2 n}{n-1}} d v\right)^{1-(1 / n)} \leq K(n) \int_{M}\left(2|f||d f|+|H| f^{2}\right) d v \\
& \quad \leq 2 K(n)\left(\int_{M} f^{2} d v\right)^{1 / 2}\left(\int_{M}|d f|^{2} d v\right)^{1 / 2}+K(n)\|H\|_{\infty} \int_{M} f^{2} d v \\
& \quad=K(n)\left(\int_{M} f^{2} d v\right)^{1 / 2}\left(2\left(\int_{M}|d f|^{2} d v\right)^{1 / 2}+\|H\|_{\infty}\left(\int_{M} f^{2} d v\right)^{1 / 2}\right)
\end{aligned}
$$


where in the second inequality, we have used the Hölder inequality. Using it again, by assuming that $V(M)=1$, we have

$$
\left(\int_{M} f^{2} d v\right)^{1 / 2} \leq\left(\int_{M} f^{\frac{2 n}{n-1}} d v\right)^{\frac{n-1}{2 n}}
$$

And finally, we obtain

$$
\|f\|_{\frac{2 n}{n-1}} \leq K(n)\left(2\|d f\|_{2}+\|H\|_{\infty}\|f\|_{2}\right) .
$$

For $k \geq 2, \xi^{k}$ is smooth and we apply the above inequality to $f=\xi^{k}$. Then we get

$$
\begin{aligned}
\|\xi\|_{\frac{2 k n}{n-1} \leq}^{k} K(n)\left[2\left(\int_{M}\left|d \xi^{k}\right|^{2} d v\right)^{1 / 2}+\|H\|_{\infty}\left(\int_{M} \xi^{2 k} d v\right)^{1 / 2}\right] \\
\leq K(n)\left[2 ( \frac { k ^ { 2 } } { 2 ( k - 1 ) } ) ^ { 1 / 2 } \left(\left(A_{1}+k A_{2}\right) \int_{M} \xi^{2 k-r} d v\right.\right. \\
\left.\left.+\left(B_{1}+k B_{2}\right) \int_{M} \xi^{2 k-s} d v\right)^{1 / 2}+\|H\|_{\infty}\left(\int_{M} \xi^{2 k} d v\right)^{1 / 2}\right] \\
\leq K(n)\left[2 ( \frac { k ^ { 2 } } { 2 ( k - 1 ) } ) ^ { 1 / 2 } \left(\left(A_{1}+k A_{2}\right)\|\xi\|_{\infty}^{2-r}\right.\right. \\
\left.\left.\quad+\left(B_{1}+k B_{2}\right)\|\xi\|_{\infty}^{2-s}\right)^{1 / 2}\|\xi\|_{2 k-2}^{k-1}+\|H\|_{\infty}\|\xi\|_{\infty}\|\xi\|_{2 k-2}^{k-1}\right] \\
\leq K(n)\left[2\left(\frac{k^{2}}{2(k-1)}\right)^{1 / 2}\left(\frac{A_{1}+k A_{2}}{\left.\|\xi\|_{\infty}^{r}+\frac{B_{1}+k B_{2}}{\|\xi\|_{\infty}^{s}}\right)^{1 / 2}}+\|H\|_{\infty}\right]\|\xi\|_{\infty}\|\xi\|_{2 k-2}^{k-1}\right. \\
\leq K(n)\left[2\left(\frac{k^{2}}{2(k-1)}\right)^{1 / 2}\left(\frac{A_{1}^{1 / 2}+k^{1 / 2} A_{2}^{1 / 2}}{\|\xi\|_{\infty}^{r / 2}}+\frac{B_{1}^{1 / 2}+k^{1 / 2} B_{2}^{1 / 2}}{\|\xi\|_{\infty}^{s / 2}}\right)\right. \\
\left.+\|H\|_{\infty}\right]\|\xi\|_{\infty}\|\xi\|_{2 k-2}^{k-1} .
\end{aligned}
$$

If we assume that $\|\xi\|_{\infty}>\eta$, the last inequality becomes

$$
\begin{aligned}
\|\xi\|_{\frac{2 k n}{n-1}}^{k} \leq K(n)\left[2 ( \frac { k ^ { 2 } } { 2 ( k - 1 ) } ) ^ { 1 / 2 } \left(\frac{A_{1}^{1 / 2}+k^{1 / 2} A_{2}^{1 / 2}}{\eta^{r / 2}}\right.\right. & \left.+\frac{B_{1}^{1 / 2}+k^{1 / 2} B_{2}^{1 / 2}}{\eta^{s / 2}}\right) \\
& \left.+\|H\|_{\infty}\right]\|\xi\|_{\infty}\|\xi\|_{2 k-2}^{k-1}
\end{aligned}
$$




$$
=\left[\left(K_{1}+k^{1 / 2} K_{2}\right)\left(\frac{k^{2}}{k-1}\right)^{1 / 2}+K^{\prime}\right]\|\xi\|_{\infty}\|\xi\|_{2 k-2}^{k-1} .
$$

Now let $q=\frac{n}{n-1}>1$ and for $i \geq 0$ let $k=q^{i}+1 \geq 2$. Then

$$
\begin{aligned}
\|\xi\|_{2\left(q^{i+1}+q\right)} & \leq\left(\left(K_{1}+\left(q^{i}+1\right)^{1 / 2} K_{2}\right)\left(\frac{q^{i}+1}{q^{i / 2}}\right)+K^{\prime \prime}\right)^{\frac{1}{q^{i}+1}}\|\xi\|_{\infty}^{\frac{1}{q^{i}+1}}\|\xi\|_{2 q^{i}}^{1-\frac{1}{q^{i}+1}} \\
& \leq\left(\tilde{K} q^{i}\right)^{\frac{1}{q^{i}+1}}\|\xi\|_{\infty}^{\frac{1}{q^{i}+1}}\|\xi\|_{2 q^{i}}^{1-\frac{1}{q^{i}+1}}
\end{aligned}
$$

where $\tilde{K}=2 K_{1}+2^{3 / 2} K_{2}+K^{\prime}$. We see that $\tilde{K}$ has a finite limit when $\eta \rightarrow \infty$ and if $B_{1}>0, \tilde{K} \rightarrow \infty$ when $\|H\|_{\infty} \rightarrow \infty$ or $\eta \rightarrow 0$. Moreover the Hölder inequality gives

$$
\|\xi\|_{2 q^{i+1}} \leq\|\xi\|_{2\left(q^{i+1}+q\right)}
$$

which implies

$$
\|\xi\|_{2 q^{i+1}} \leq\left(\tilde{K} q^{i}\right)^{\frac{1}{q^{i}+1}}\|\xi\|_{\infty}^{\frac{1}{q^{i}+1}}\|\xi\|_{2 q^{i}}^{1-\frac{1}{q^{i}+1}} .
$$

Now, by iterating from 0 to $i$, we get

$$
\begin{aligned}
& \|\xi\|_{2 q^{i+1}} \\
& \leq \tilde{K}^{\left(1-\prod_{k=i-j}^{i}\left(1-\frac{1}{q^{k}+1}\right)\right)} q^{\sum_{k=i-j}^{i} \frac{k}{q^{k}+1}\|\xi\|_{\infty}^{\left(1-\prod_{k=i-j}^{i}\left(1-\frac{1}{q^{k}+1}\right)\right)}\|\xi\|_{2 q^{i-j}} \prod_{k=i-j}^{i}\left(1-\frac{1}{q^{k}+1}\right)} \\
& \leq \tilde{K}^{\left(1-\prod_{k=0}^{i}\left(1-\frac{1}{q^{k}+1}\right)\right)} q^{\sum_{k=0}^{i} \frac{k}{q^{k}+1}\|\xi\|_{\infty}^{\left(1-\prod_{k=0}^{i}\left(1-\frac{1}{q^{k}+1}\right)\right)}\|\xi\|_{2} \prod_{k=0}^{i}\left(1-\frac{1}{q^{k}+1}\right) .} \\
& \text { Let } \alpha=\sum_{k=0}^{\infty} \frac{k}{q^{k}+1} \text { and } \beta=\prod_{k=0}^{\infty}\left(1-\frac{1}{q^{k}+1}\right)=\prod_{k=0}^{\infty}\left(\frac{1}{1+(1 / q)^{k}}\right) \text {. Then } \\
& \|\xi\|_{\infty} \leq \tilde{K}^{1-\beta} q^{\alpha}\|\xi\|_{\infty}^{(1-\beta)}\|\xi\|_{2}^{\beta}
\end{aligned}
$$

and finally

$$
\|\xi\|_{\infty} \leq L\|\xi\|_{2}
$$

where $L=\tilde{K}^{\frac{1-\beta}{\beta}} q^{\alpha / \beta}$ is a constant depending only on $n, A_{1}, A_{2}, B_{1}, B_{2},\|H\|_{\infty}$ and $\eta$. From classical methods we show that $\beta \in\left[e^{-n}, e^{-n / 2}\right]$. In particular, $0<\beta<1$ and we deduce that $L$ is bounded when $\eta \rightarrow \infty$ and $L \rightarrow \infty$ when $\|H\|_{\infty} \rightarrow \infty$ or $\eta \rightarrow 0$ with $B_{1}>0$.

Remark. In [12] and [13] Shihohama and Xu have proved that if $\left(M^{n}, g\right)$ is a compact $n$-dimensional Riemannian manifold without boundary isometrically immersed in 
$\mathbb{R}^{n+1}$ and if $\int_{M}\left(|B|^{2}-n|H|^{2}\right)<D_{n}$ where $D_{n}$ is a constant depending on $n$, then all Betti numbers are zero. For $n=2, D_{2}=4 \pi$, and it follows that if

$$
\int_{M}|B|^{2} d v-4 \pi<\lambda_{1}(M) V(M)
$$

then we deduce from the Reilly inequality $\lambda_{1}(M) V(M) \leq 2 \int_{M} H^{2} d v$ that $\int_{M}\left(|B|^{2}-2|H|^{2}\right) d v<4 \pi$ and by the result of Shihohama and $\mathrm{Xu} M$ is diffeomorphic to $\mathbb{S}^{2}$.

\section{References}

[1] D. Burago, V. A. Zalgaller, Geometric Inequalities. Grundlehren Math. Wiss. 285, Springer-Verlag, Berlin 1988. Zbl 0633.53002 MR 0936419

[2] T. H. Colding, Shape of manifolds with positive Ricci curvature. Invent. Math. 124 (1996), 175-191. Zbl 0871.53027 MR 1369414

[3] T. H. Colding, Large manifolds with positive Ricci curvature. Invent. Math. 124 (1996), 193-214. Zbl 0871.53028 MR 1369415

[4] C. B. Croke, An eigenvalue Pinching Theorem. Invent. Math. 68 (1982), 253-256. Zbl 0505.53018 MR 0666162

[5] J. H. Eschenburg, Diameter, volume and topology for positive Ricci curvature. J. Differential Geom. 33 (1991), 743-747. Zbl 0724.53026 MR 1100210

[6] D. Hoffman, J. Spruck, Sobolev and isoperimetric inequalities for Riemannian submanifolds. Comm. Pure Appl. Math. 27 (1974), 715-727. Zbl 0295.53025 MR 0365424

[7] D. Hoffman, J. Spruck, Sobolev and isoperimetric inequalities for Riemannian submanifolds. Erratum Comm. Pure Appl. Math. 28(1975), 765-766. Zbl 0327.53035 MR 0397625

[8] Ilias S., Un nouveau résultat de pincement de la première valeur propre du Laplacien et conjecture du diamètre pincé. Ann. Inst. Fourier 43 (3) (1993), 843-863. Zbl 0783.53024 MR 1242618

[9] A. Lichnerowicz, Géométrie des groupes de transformations. Travaux et Recherches Mathématiques III, Dunod, Paris 1958. Zbl 0096.16001 MR 0124009

[10] P. Petersen, On eigenvalue pinching in positive Ricci curvature. Invent. Math. 138 (1999), 1-21. Zbl 0988.53011 MR 1714334

[11] R. Reilly, On the first eigenvalue of the Laplacian for compact submanifolds of Euclidean space. Comment. Math. Helv. 52 (1977), 525-533. Zbl 0382.53038 MR 0482597

[12] K. Shiohama, H. Xu, Rigidity and sphere theorems for submanifolds. Kyushu J. Math. 48 (2) (1994), 291-306. Zbl 0826.53045 MR 1294532

[13] K. Shiohama, H. Xu, Rigidity and sphere theorems for submanifolds II. Kyushu J. Math. 54 (1) (2000), 103-109. Zbl 1005.53028 MR 1762795

[14] T. Takahashi, Minimal immersions of Riemannian manifolds. J. Math. Soc. Japan 18 (1966), 380-385. Zbl 0145.18601 MR 0198393 
Vol. 82 (2007)

[15] J. Y. Wu, A diameter pinching sphere theorem for positive Ricci curvature. Proc. Amer. Math. Soc. 107 (3) (1989), 797-802. Zbl 0676.53045 MR 0984822

[16] H. W. Xu, Some results on geometry of Riemannian submanifolds. Ph.D. Dissertation, Fudan University, 1990.

Received October 21, 2003; revised November 4, 2005

Bruno Colbois, Institut de Mathématiques, Université de Neuchâtel, Rue Émile Argand 11, 2007 Neuchâtel, Switzerland

E-mail: bruno.colbois@unine.ch

Jean-François Grosjean, Institut Élie Cartan (Mathématiques), Université Henri Poincaré Nancy I, B.P. 239, 54506 Vandoeuvre-les-Nancy Cedex, France

E-mail: grosjean@iecn.u-nancy.fr 